\title{
Are both symmetric and buckled dimers on Si(100) minima? Density functional and multireference perturbation theory calculations
}

\author{
Yousung Jung, Yihan Shao, Mark S. Gordon ${ }^{\star}$, Douglas J. Doren ${ }^{\S}$ and Martin Head-Gordon* \\ Department of Chemistry, University of California at Berkeley, \\ and Chemical Sciences Division, Lawrence Berkeley National Laboratory, Berkeley, CA 94720, \\ ${ }^{*}$ Department of Chemistry, Iowa State University, Ames, Iowa 50011 \\ ${ }^{\S}$ Department of Chemistry and Biochemistry, University of Delaware, Newark, Delaware 19716
}

\begin{abstract}
We report a spin-unrestricted density functional theory (DFT) solution at the symmetric dimer structure for cluster models of $\operatorname{Si}(100)$. With this solution, it is shown that the symmetric structure is a minimum on the DFT potential energy surface, although higher in energy than the buckled structure. In restricted DFT calculations the symmetric structure is a saddle point connecting the two buckled minima. To further assess the effects of electron correlation on the relative energies of symmetric versus buckled dimers on $\operatorname{Si}(100)$, multireference second order perturbation theory (MRMP2) calculations are performed on these DFT optimized minima. The symmetric structure is predicted to be lower in energy than the buckled structure via MRMP2, while the reverse order is found by DFT. The implications for recent experimental interpretations are discussed.
\end{abstract}

*Corresponding author email: mhg@bastille.cchem.berkeley.edu 


\section{Introduction}

The silicon (100) surface is one of the most extensively studied systems in surface science, both experimentally and theoretically, due to its technological importance in device fabrication. ${ }^{1-25}$ It undergoes surface reconstruction when cleaved, in which the surface silicon atoms dimerize to form an energetically more favorable sigma bond..$^{1-5}$ However, whether these surface dimers are symmetric or buckled (or asymmetric), due to the two remaining dangling bonds, is still a question that continues to be discussed. Part of the reason for this is in the very flat nature of the potential energy surface of Si(100) along buckling coordinates. Because of this delicacy, small variations in experimental conditions or use of different theoretical methods have given contradictory results on the structure of $\operatorname{Si}(100)$.

In experiments, there have been numerous indications from spectroscopy, ${ }^{6}$ diffraction, ${ }^{7}$ and scanning tunneling microscopy (STM), ${ }^{1-3,5,9-12}$ that the dimers are intrinsically buckled above $100 \mathrm{~K}$. The dominant appearance of the symmetric dimers in STM images at room temperature was ascribed to the dynamic flipping motion of the buckled dimers. The buckled dimers are observed predominantly at low temperatures $(110-120 \mathrm{~K}) .{ }^{5,8}$ However, recent STM studies reported contradictory results upon further cooling (below $65 \mathrm{~K}$ ). Kondo et al. predicted that the symmetric dimer is the ground state, ${ }^{8}$ whereas others concluded that reappearance of the symmetric dimers at very low temperatures results from a reduced flipping barrier. ${ }^{9}$ This low temperature phase transition (around $40 \mathrm{~K}$ ) was also observed by several other groups using STM, synchrotron-radiation photoelectron spectroscopy, and low-energy electron diffraction (LEED). ${ }^{10-12}$ However, these later experiments disagree with the stable symmetric dimer inferred at $0 \mathrm{~K}$ by Kondo et al. Instead, they suggest an asymmetric $p(2 \times 1)$ phase, a $p(2 \times 2)$ phase, or a disordered $p(2 \times 1)$ phase due to the dynamic flip-flop motion of the buckled dimers at low $(<40 \mathrm{~K})$ temperatures. 
In particular, Lay et al. observed asymmetric dimers down to $40 \mathrm{~K}$ using synchrotron-radiation photoelectron spectroscopy, and proposed that this $p(2 \times 1)$ structure is either composed of static buckled dimers or due to an artifact of local tip-dimer interactions in the STM. ${ }^{10}$ They excluded the possibility of a dynamic flip-flop motion. On the other hand, Hata et al. observed exclusively the $p(2 \times 2)$ phase below $40 \mathrm{~K}$ on $n$-type substrates, and provided evidence that the $p(2 \times 1)$ symmetric dimers can also be caused by the STM tip-induced flip-flop motion. ${ }^{11}$ More recent LEED data confirmed a phase transition from an ordered $c(4 \times 2)$ to the disordered $p(2 \times 1)$ phase at around $40 \mathrm{~K}$. The authors suggested quantum coherence between the degenerate buckled states as a mechanism for the reappearing $p(2 \times 1)$ structure at low temperatures. ${ }^{12}$

With respect to calculations, which are the focus of this paper, density functional theory (DFT) and quantum Monte Carlo (QMC) calculations predict the buckled structure to be the minimum, ${ }^{17-22}$ whereas high level multireference wave function based methods predicted the symmetric structure to be the minimum. ${ }^{13-17}$ The first uncertainty in these calculations is the validity of the surface model, which is either a hydrogen-terminated cluster or a periodic slab model containing several layers. ${ }^{23}$ The simplest cluster model for $\mathrm{Si}(100)$ is $\mathrm{Si}_{9} \mathrm{H}_{12}$, which has a single surface dimer. One can then increase the number of dimers systematically by adding more dimer units along the same row (Figure 1), and study the

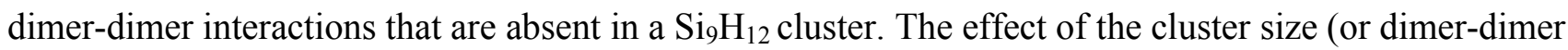
interactions) on the structure of $\mathrm{Si}(100)$ has been investigated, and again, DFT and QMC emphasized inter-dimer interactions that favor the buckled structure, ${ }^{19,21}$ whereas the multiconfiguration self consistent field (MCSCF) methods and multireference second-order perturbation theory (MRMP2) predicted these effects to be small and the lowest energy structure to remain symmetric. ${ }^{16}$

The second source of uncertainty in the calculations is the adequacy with which electron correlation is treated. Electron correlation is often separated into dynamic and non-dynamic (or static) components, although there is no unique way of dividing them. Roughly, dynamic correlation is the electron correlation due to instantaneous repulsive electronic motions, and non-dynamic correlation is the electron correlation arising from near-degeneracies in the wave function. Static correlation in 
$\mathrm{Si}(100)$ is associated with bond-antibond correlations arising because of the relatively small gap between the HOMO $(\pi)$ and LUMO $\left(\pi^{*}\right)$ in each dimer bond (reflecting their origin as dangling bonds). Sufficient inclusion of electron correlation in the silicon surface is critical for determining the surface structure because of the low frequency buckling vibrations in $\operatorname{Si}(100)$.

For a given cluster model, DFT and MCSCF emphasize somewhat different electron correlation effects. DFT incorporates some dynamic correlation via a parameterized correlation functional, whereas MCSCF emphasizes static correlation when the active space is properly chosen, and essentially neglects dynamic correlation. The controversy between DFT and MCSCF for the $\mathrm{Si}(100)$ problem is probably largely related to this difference in the manner in which correlation is treated. There have been some recent efforts to explicitly include both correlation effects, including recent diffusion Monte Carlo $(\mathrm{DMC})^{24}$ and MRMP2 calculations ${ }^{16}$ using large (2-, 3- and 5-dimer) clusters. However, these two approaches also yield contradictory results. DMC predicts the buckled structure to be the lower in energy, whereas MRMP2 predicts the symmetric structure to be lower in energy. Use of DFT optimized geometries and single configuration trial functions for 2-and 3-dimer clusters in DMC calculations, and use of MCSCF geometries in MRMP2 calculations were proposed as possible sources of the contradiction, as discussed in more detail in reference 16. However, the basic inconsistency between QMC/DFT and MRMP2/MCSCF still remains.

In this paper, we carefully (re)examine the DFT potential energy surface for $\mathrm{Si}(100)$ using cluster models including up to four dimers. To the best of our knowledge, no spin-unrestricted solution was found for the symmetric structure in the previous DFT studies using cluster models, ${ }^{22}$ although a spin-polarized DFT solution with the BPW91 functional was reported using the slab model hinting that the symmetric structure could be a minimum. ${ }^{18}$ It is also noted that the importance of including spin considerations (arrangements) for a correct description of the symmetric dimers (and the relative stability between the symmetric and buckled dimers) at the model Hamiltonian and Hartree-Fock levels has been discussed elsewhere. ${ }^{25}$ 
Our calculations show that there is a lower energy spin-unrestricted DFT solution for the symmetric cluster. In the single-dimer model, the stabilization of this state is quite small $(0.16 \mathrm{kcal} / \mathrm{mol})$, but it is significant for larger clusters $(2 \sim 3 \mathrm{kcal} / \mathrm{mol})$. Moreover, this symmetric structure in all the cluster models studied here is shown to be a true minimum on the DFT potential energy surface, although the buckled structure is still the DFT global minimum (at least with the functional used here). This is significant since the symmetric structure is a saddle point connecting the two buckled minima in unstable restricted DFT models. We also note that, although in a previous DFT study certain functionals (BP and BLYP) with the constrained single-dimer cluster predicted the symmetric dimer to be a (flat) minimum, the energy profile shown for the multiple-dimer models implied it to be a saddle point. ${ }^{21}$ Whether or not the correct unrestricted solution was used in this study was not clear.

To examine the effect of explicitly including both dynamical and non-dynamical correlations, we also performed MRMP2 calculations on these DFT minima. This is different from the previous QMC and MRMP2 calculations in that the geometries used in this study are well balanced, with both structures being minima on the DFT potential surface. Detailed results and their implications are discussed in the Results section. 


\section{Theoretical methods}

$\mathrm{Si}_{9} \mathrm{H}_{12}, \mathrm{Si}_{15} \mathrm{H}_{16}, \mathrm{Si}_{21} \mathrm{H}_{20}$, and $\mathrm{Si}_{27} \mathrm{H}_{24}$ clusters were used as model surfaces, which correspond to 1-, 2-, 3-, and 4-dimer clusters, respectively. 3- and 4-dimer clusters are the smallest cluster models in which edge effects can be minimized and hopefully eliminated. The largest (4-dimer) cluster model used in this study is shown in Figure 1.

Spin contamination in $\mathrm{DFT}^{26}$ is not strictly meaningful, unlike in Hartree-Fock (HF) theory. This is because the Slater single determinant of Kohn-Sham (KS) orbitals that produces the correct density is clearly not the correct wave function. This makes the use of the reference DFT "wave function" to calculate $<S^{2}>$ not theoretically rigorous. Therefore, in performing DFT calculations, spin-unrestricted DFT should be used whenever it is possible to find a spin-unrestricted lower energy solution. ${ }^{27}$ Spinunrestricted KS theory with the B3LYP functional ${ }^{28}$ was used with the 6-31G(d) basis, ${ }^{29}$ namely, (U)B3LYP/6-31G(d), for all DFT calculations. Unrestricted solutions were found by mixing the restricted alpha HOMO and LUMO to break spin symmetry, followed by minimization via an orbital gradient search method. ${ }^{30}$

In employing cluster models for the surface reconstruction, various structural constraints can be used to mimic the real surface. For example, if the atoms several layers below the surface do not change much from their bulk positions upon surface reconstruction, constraining them to be fixed at their bulk positions can be an effective way of using cluster models. A density functional study by Yang and Kang, ${ }^{20}$ however, showed that the energetics and surface structures are quite sensitive to the use of different geometry constraints in cluster models. More recently, moreover, large cluster generalized valence bond-perfect pairing (GVB-PP) calculations established that the surface relaxation and reconstruction are propagated several layers into the bulk, and that the imposition of geometry constraints can result in nonphysical structural predictions for the $\mathrm{Si}(100)$ surface. ${ }^{14}$

Following that conclusion, for this work, no structural constraints were imposed for geometry optimizations, except for the use of molecular point group symmetry. $C_{2}$ (or $C_{s}$ for $\mathrm{Si}_{9} \mathrm{H}_{12}$ and $\mathrm{Si}_{21} \mathrm{H}_{20}$ ) 
and $C_{2 v}$ symmetries were used for the buckled and symmetric structures, respectively. The analytic hessian in coordinate space (second derivative of energy with respect to the $3 \mathrm{~N}$ atomic displacements, where $\mathrm{N}$ is the number of atoms in a system) was calculated to characterize the stationary points found from geometry optimizations.

Just as the hessian in real space can tell us if the stationary point located is a minimum or a saddle point, calculating the hessian in orbital space (second derivative of energy with respect to the orbital rotations) can indicate if the SCF solution found is a real minimum or a saddle point in orbital space. ${ }^{31}$ In other words, the stability of the SCF solution can be analyzed by calculating the hessian in orbital space. All DFT calculations were initially performed with restricted orbitals, and were then tested for external stability (with respect to spin symmetry breaking). Unstable restricted solutions were then reoptimized after breaking spin symmetry to yield unrestricted solutions with lower energy. Their stability was then verified.

MRMP2 is a method that corrects a multiconfigurational reference (MR) wave function (that captures non-dynamic correlation) with second order Møller-Plesset (MP2) perturbation theory (that captures dynamic correlation). In principle this should provide a balanced treatment of buckled versus symmetric dimers, and we therefore performed single point MRMP2 calculations on the (U)B3LYP/631G(d) optimized geometries. The reference wave function includes all excitations in the space of one bonding and one antibonding orbital for each dimer (i.e. 2 electrons in 2 orbitals for $\mathrm{Si}_{9} \mathrm{H}_{12}$, 4 -in-4 for $\mathrm{Si}_{15} \mathrm{H}_{16}$, and 6-in-6 $\mathrm{Si}_{21} \mathrm{H}_{20}$ ). The 6-31G(d) basis was used, with additional tests for basis set extension effects as described in the Results section.

All DFT calculations were carried out using Q-CHEM, ${ }^{32}$ and MRMP2 calculations were carried out using GAMESS. ${ }^{33}$ 


\section{Results and discussion}

Density functional theory (DFT). Tables 1 and 2 summarize important structural parameters (buckling angles, dimer bond lengths, and spin densities at dimer sites) and $<S^{2}>$ values for the buckled and symmetric structures, respectively, as a function of the number of dimers in a cluster. We define the buckling angle as in Figure 2 although there is no unique single definition.

Careful examination of the $\mathrm{Si}_{9} \mathrm{H}_{12}$ cluster revealed that there $i s$ a spin-unrestricted lower energy solution for the symmetric structure $\left(C_{2 v}\right)$, and it indeed is a true minimum on the (U)B3LYP potential energy surface. Although the effect on energy is small in this model with the unrestricted solution being only $0.16 \mathrm{kcal} / \mathrm{mol}$ lower than the restricted solution (last column in Table 2), this result is significant in that the symmetric structure is a transition state connecting the two buckled minima in restricted DFT models. We also note that, in the previous DFT cluster studies that searched for instabilities, ${ }^{22}$ no spinunrestricted solution was found, presumably due to this small energy difference between the solutions for the single-dimer model. ${ }^{34}$

Stability analyses were performed for both spin-restricted and unrestricted solutions at the symmetric structure. As expected from the existence of a lower energy spin-unrestricted solution, the spin-restricted solution at the symmetric structure is found to be unstable in orbital space (1 negative eigenvalue for the 1-dimer cluster), whereas a spin-unrestricted solution is stable, or a true minimum (0 negative eigenvalues). ${ }^{34}$ On this basis, we conclude that the previously reported DFT cluster results implying that the symmetric structure was a saddle point were based on the use of higher energy unstable restricted SCF solutions. In fact, our finding of a spin-unrestricted solution for the symmetric structure is consistent with the recent spin polarized DFT study, in which the authors found spin polarized lower energy solutions at geometries with small buckling angles by using a slab model although it was not conclusively established to be a minimum. ${ }^{18,25}$ 
Finding a spin-unrestricted lower energy solution has a dramatic effect on the 1-dimer cluster. Geometry optimization of the buckled structure $\left(C_{s}\right)$ with unrestricted orbitals restores the (almost) symmetric structure with a buckling angle of $0.4^{\circ}$. The energy difference $\left(\Delta \mathrm{E}=\mathrm{E}_{\mathrm{sym}}-\mathrm{E}_{\text {buck }}\right)$ between the symmetric and buckled structures is $0.05 \mathrm{kcal} / \mathrm{mol}$, very marginally favoring the buckled structure. It is also noted that both the symmetric and buckled structures in the $\mathrm{Si}_{9} \mathrm{H}_{12}$ cluster have spin-unrestricted solutions, as can be seen in significant spin densities at the dimer silicons, $<S^{2}>$ values, and (small but non-zero) energy differences between the spin-restricted and -unrestricted solutions, $\Delta E(R \rightarrow U)$, in Tables 1 and 2.

Of course, $\mathrm{Si}_{9} \mathrm{H}_{12}$ is too small a cluster to represent real surface in which dimers can interact with each other, as pointed out many times previously. To examine the effects of dimer-dimer interactions on buckling, we therefore systematically investigated 2-, 3-, and 4-dimer clusters. Three major conclusions can be drawn from these larger cluster calculations.

First, the symmetric structures $\left(C_{2 v}\right)$ in all cluster models have spin-unrestricted solutions and are true energy minima. Positive definite hessians in both coordinate and orbital space establish these results. As can be seen in Table 2, the unrestricted solutions at the symmetric structure are lower than the restricted solutions by $2.0,3.4$, and $2.8 \mathrm{kcal} / \mathrm{mol}$ in energy for the 2-, 3- and 4-dimer models, respectively. These energy differences, $\Delta \mathrm{E}(\mathrm{R} \rightarrow \mathrm{U})$, are significant compared to the 1-dimer case.

Second, adding another dimer to a single dimer cluster has a dramatic effect on the buckled structure, in which the buckling angle increases substantially from $0.5^{\circ}$ to $13.1^{\circ}$ (Table 1 ). Moreover, the restricted solution is stable for the buckled structures in these multiple-dimer models. This is also consistent with the LUMO occupation numbers in CASSCF single-point calculations on the DFT minima changing from $\sim 0.35$ at the symmetric structure to 0.07 at the buckled structure, for the middle dimer of the 3-dimer cluster. This suggests that including inter-dimer interaction is critical in determining the correct buckled structure, at least within the DFT method. It should be noted that, by contrast, in MCSCF, adding more dimers to the model does not change the qualitative result, with the symmetric structure consistently favored. ${ }^{16}$ 
Third, detailed structural parameters for the middle dimer (i.e., buckling angles, dimer bond lengths, and spin densities at dimer sites), in both the buckled and symmetric structures, appear to converge approximately at the 3 -dimer cluster. This result suggests that the screening of edge effects is important when using clusters to model the $\operatorname{Si}(100)$ surface. Therefore, to obtain reasonable surface structures for $\mathrm{Si}(100)$ using cluster models, clusters containing at least three dimers should be used. It is also worthwhile to note that, based on the dimer site spin densities given in Table 2, as the cluster size increases, the extent of diradical character of the middle dimer for the symmetric structures increases. This trend in diradical character is also suggested by the trend in bond length (Table 2). However, this trend is not observed in CASSCF calculations for symmetric 1-5 dimer clusters in which the natural orbital occupation numbers are found to be $\sim 1.65$ (HOMO) and $\sim 0.35$ (LUMO) for all structures that were studied. ${ }^{16}$ The dimer bond lengths did not show any particular trend either in these CASSCF calculations. ${ }^{16}$

Table 3 summarizes the energy difference between the symmetric and buckled structures per dimer for various cluster sizes, with a positive sign meaning that the buckled structure is lower in energy. As earlier DFT studies proposed, $\triangle \mathrm{E}$ per dimer increases with the number of dimers in the cluster model, due to inter-dimer interactions. Clusters including more dimers favor the buckled structure to avoid dimer-dimer repulsion (which is maximal in the symmetric configuration), and to optimize electrostatic interactions between dimers. In fact, for a 3-dimer cluster, the energy gain by buckling compared to the symmetric structure was estimated to be $8.78 \mathrm{kcal} / \mathrm{mol}$ in the previous MCSCF study, although MCSCF still favored the symmetric structure overall. ${ }^{16}$

Due to the demanding computational cost, clusters including more than four dimers were not investigated in this work. The trend in Table 3, however, suggests that the converged value for $\Delta \mathrm{E}$ per dimer will not be far from the one obtained with a 4-dimer cluster. Comparison with the previous DFT/BPW91 results ${ }^{17}$ in Table 3 also shows that our values in all the cluster models are somewhat smaller than the previously reported numbers. These differences (decreases) are due to the lower energy spin-unrestricted solutions found for the symmetric structures in this study. 
Multireference perturbation theory (MRMP2). We applied the MRMP2 method to the DFT optimized minima to get relative energetics that include explicit treatment of both dynamic and nondynamic correlation effects. The symmetric structure is predicted to be lower in energy than the buckled structure by $3.35 \mathrm{kcal} / \mathrm{mol}$ per dimer for a 2-dimer cluster, and $4.01 \mathrm{kcal} / \mathrm{mol}$ per dimer for a 3-dimer cluster, as summarized in Table 3. This is the opposite of the DFT results elaborated above! We note that for the single dimer, since there is virtually no difference in geometry between the symmetric and buckled dimers (Table 1 and 2), they are almost equal in energy at the CASSCF and MRMP2 levels.

Since these energy differences are relatively small even for 2- and 3-dimer clusters, to assess the basis set effect, we also carried out the same MRMP2 calculations for a 2-dimer cluster using a bigger basis. This consists of $6-31+\mathrm{G}(3 \mathrm{df})$ for the dimer silicon atoms and $6-31 \mathrm{G}(\mathrm{d})$ for the rest of the cluster. The result is essentially the same as that obtained using the 6-31G(d) basis for the whole cluster, favoring the symmetric structure by $3.09 \mathrm{kcal} / \mathrm{mol}$ per dimer. Therefore, the MRMP2 results with the 6$31 \mathrm{G}(\mathrm{d})$ basis in Table 3 are apparently not biased by using this relatively small basis.

There are two important conclusions from these results. First, as suggested in the previous DFT studies, ${ }^{22,24}$ our results also indicate that the buckled structure benefits more from the inclusion of dynamic correlation than the symmetric structure. This is evidenced by the fact that, for example for the 2-dimer cluster, the (MR)MP2 correction to CASSCF lowers $\Delta \mathrm{E} /$ dimer from -6.71 to $-3.35 \mathrm{kcal} / \mathrm{mol}$ in Table 3, stabilizing the buckled structure by $3.36 \mathrm{kcal} / \mathrm{mol}$ per dimer as a result of including dynamic correlation.

Second, however, the effect of dynamic correlation is not sufficient for the buckled structure to become a global minimum overall, because this additional energy gain in the buckled structure from including dynamic correlation is more than offset by the importance of non-dynamic correlation in the symmetric structure. This implies that an adequate treatment of the diradicaloid dimers at the symmetric structure via a multireference wave function is crucial in determining the delicate energetics of the $\operatorname{Si}(100)$ surface. 
Discussion. Our finding that buckled structures are the global energy minima on the DFT potential surface, while the symmetric dimers are metastable minima, appears to be consistent with some recent experiments that support the presence of buckled dimers at low temperatures. ${ }^{9-12}$ However, the question of whether the metastable symmetric state might be observed directly makes the height of the conversion barrier from the symmetric to buckled dimers (or vice versa) interesting, since this will determine the conversion rate at (finite) low temperatures, if the symmetric dimers are indeed stable.

In fact, the barrier from the symmetric to buckled dimers was estimated to be less than 0.2 $\mathrm{kcal} / \mathrm{mol}$ per dimer in Ref. 18. This is in good agreement with our preliminary result of $\sim 0.1 \mathrm{kcal} / \mathrm{mol}$ per dimer using the 2-dimer cluster at the UB3LYP/6-31G(d) level of theory. These results indicate that, at least at the DFT level, the higher symmetric minimum is extremely shallow, and is therefore not likely to be seen as a static state in experiments due to the rapid thermal conversion at temperatures above $0 \mathrm{~K}$. This DFT result, however, needs some improvements to make a more concrete connection to the recent experiments. Given the critical importance of electron correlation in $\mathrm{Si}(100)$ as described in the previous section, geometry optimizations with higher level methods such as MRMP2 or DMC are essential to obtain more reliable quantitative (or possibly even qualitative) results for this conversion. It is also possible to imagine a mixed configuration (some buckled plus some symmetric dimers) in a local environment, which presumably will be higher in energy than a pure configuration (buckled or symmetric), but could be entropically favored. For this, at least 3-dimer cluster models are required. Depending upon the balance of entropic gain and energetic cost, as well as the kinetic barrier, such a configuration may also be thermally accessible in experiments. These calculations are in progress, and may provide useful connections to the low temperature experiments.

Finally, the problem of establishing the preferred ordering of the symmetric and buckled dimers from electronic structure calculations (still) requires additional work. There remain a number of unresolved issues concerning the discrepancy between QMC/DFT which favor buckled structures, and the highest level wave function calculations reported here, MRMP2, which favors symmetric dimers. 
Does MRMP2 predict two different local minima, like DFT, or just a single minimum like CASSCF? If MRMP2 indeed does predict the two local minima (buckled and symmetric), what is the barrier height for the conversion at this level of theory? Is the MP2 treatment of dynamic correlation in MRMP2 adequate? What relative energies would be obtained by quantum Monte Carlo (QMC) calculations using the new (symmetric) geometries reported here, or by performing QMC geometry optimizations? How strongly would the QMC results be affected by using a small MCSCF trial function instead of a single determinant? Answers to these questions should more fully clarify the potential energy surface of these cluster models of $\operatorname{Si}(100)$.

\section{Conclusions}

(1) Spin-unrestricted density functional theory (DFT) with the B3LYP functional for cluster models of $\operatorname{Si}(100)$ yields a metastable local minimum at the symmetric dimer structure, in addition to the global minimum, buckled dimer geometry. This is in contrast with the spin-restricted DFT calculations that predict the symmetric structure to be a saddle connecting the two buckled minima. Relative energies of these two structures were assessed by multireference perturbation theory (MRMP2) as well as by DFT. The results are contradictory. In DFT, the buckled structure is predicted to be more stable than the symmetric structure by $1.54 \mathrm{kcal} / \mathrm{mol}$ per dimer using a 4-dimer cluster, while in MRMP2, the symmetric structure is predicted to be more stable than the buckled structure by $4.1 \mathrm{kcal} / \mathrm{mol}$ per dimer using a 3-dimer cluster. It remains to be seen how geometry optimization with MRMP2 affects this result, and whether it also yields two distinct local minima.

(3) Preliminary results for the conversion barrier from the symmetric to buckled dimers indicates that the symmetric minimum is extremely shallow and therefore its 
conversion to the lower energy buckled minimum is almost barrierless, at least at the DFT level of theory. This rapid thermal conversion of the symmetric dimers may prevent them from being seen as static states in experiments. This DFT result, however, needs to be validated or refined using higher level methods such as MRMP2 or DMC with larger cluster models for more definitive connection to the experiments. Cluster size and basis set will also need to be carefully tested for converged results. This is in progress. 
Acknowledgement. This work was supported by the Department of Energy, SciDAC Computational Chemistry Program (Grant No. DE-FG02-01ER403301), and by a grant of supercomputer time from NERSC. The authors are grateful to Professor Chris Cramer and Professor Ken Jordan for enlightening discussions. We also thank one of the referees for informing us of important recent experimental papers.

\section{References}

1. H. Neergaard and J. T. Yates, Chem. Rev. 95, 1589 (1995)

2. R. M. Tromp, R. J. Hamers, and H, E. Demuth, Phys. Rev. Lett. 55, 1303 (1985); R. J. Hamers, R. M. Tromp, J. E. Demuth, Phys. Rev. B 34, 5343 (1986); R. J. Hamers, Ph. Avouris, and F. Bozso ibid. 59, 2071 (1987); R. J. Hamers, Rh. Avouris, and F. Bozso, J. Vac. Sci. Technol. A 6, 508 (1988); R.J. Hamers and Y. Wang, Chem. Rev. 96,1261 (1996)

3. D. Badt, H. Wengelnik, H. Neddermeyer, J. Vac. Sci. Technol. B 12, 2015 (1994)

4. W. A. Goddard III and T. C. McGill, J. Vac. Sci. Technol. 16, 1308 (1979)

5. R. A. Wolkow, Phys. Rev. Lett. 68, 2636 (1992)

6. F.J. Himpsel, P. Heimann, T.-C. Chang and D.E. Eastman, Phys. Rev Lett. 45, 1112 (1980); L.S.O Johansson, R.I.G. Uhrberg, P. Martensson and G.V. Hansson, Phys. Rev. B 42, 1305 (1990); G.K. Wertheim, D.M. Riffe, J.E. Rowe and P.H. Citrin, Phys, Rev. Lett. 67, 120 (1991); E. Landemark, C.J. Karlsson, Y.-C. Chao and R.I.G. Uhrberg, Phys. Rev. Lett. 69, 1588 (1992); A. I. Shkrebtii and R. Del Sole, Phys. Rev. Lett. 70, 2645 (1993)

7. B. W. Holland, C.B. Duke and A. Paton, Surf. Sci. 140, L269 (1984); N. Jedrecy, M. SauvageSimkin, R. Pinchaux, J. Massies, N. Greiser and V.H. Etgens, Surf. Sci. 230, 197 (1990); G. Jayaram, P. Xu and L.D. Marks, Phys. Rev. Lett 71, 3489 (1993); H. Over, J. Wasserfall, W. Ranke, C. Ambiatello, R. Sawitzki, D. Wolf and W. Moritz, Phys. Rev. Lett. 55, 4731 (1997); E.L. Bullock, R. Gunnella, L. Patthey, T. Abukawa, S. Kono, C.R. Natoli and L.S.O. Johansson, Phys. Rev. Lett. 74, 2756 (1995)

8. Y. Kondo, T. Amakusa, M. Iwatsuki, H. Tokumoto, Surf. Sci. 453, L318 (2000) 
9. T. Yokoyma and K. Takayanagi, Phys. Rev. B 61, R5078 (2000); T. Mitsui and K. Takayanagi, Phys. Rev. B 62, R16251 (2000)

10. G. Le Lay, A. Criecenti, C. Ottaviani, P. Perfetti, T. Tanikawa, I. Matsuda, S. Hasegawa, Phys. Rev. B 66, 153317 (2002)

11. K. Hata, Y. Sainoo, H. Shigekawa, Phys. Rev. Lett. 86, 3084 (2001); K. Hata, S. Yoshida, H. Shigekawa, Phys. Rev. Lett. 89, 286104 (2002)

12. M. Matsumoto, K. Fukutani, T. Okano, Phys. Rev. Lett. 90, 106103 (2003)

13. A. Redondo, and W. A. Goddard III, J. Vac. Sci. Technol. 21, 344 (1982)

14. J. Shoemaker, J. W. Burggraf, and M. S. Gordon, J. Chem. Phys. 112, 2994 (2000)

15. M. S. Gordon, J. R. Shoemaker, and L. W. Burggraf, J. Chem. Phys. 113, 9355 (2000)

16. Y. Jung, Y. Akinaga, K. D. Jordan, and M. S. Gordon, Theor. Chem. Acc. In press (published online) (2003)

17. P. Bokes, I. Stich, L. Mitas, Chem. Phys. Lett. 362, 559 (2002)

18. O. Paz, A. J.R. da Silva, J. J. Saenz, E. Artacho, Surf. Sci. 482-485, 458 (2001)

19. R. Konecny, and D. J. Doren, J. Chem. Phys. 106, 2426 (1997)

20. C. Yang, and H. C. Kang, J. Chem. Phys. 110, 11029 (1999)

21. E. Penev, P. Kratzer, and M. Scheffler, J. Chem. Phys. 110, 3986 (1999)

22. J. S. Hess, and D. J. Doren, J. Chem. Phys. 113, 9353 (2000)

23. J. A. Steckel, T. Phung, K. D. Jordan, P. Nachtigall, J. Phys. Chem. B 105, 4031 (2001)

24. S. B. Healy, C. Filippi, P. Kratzer, E. Penev, and M. Sheffler, Phys. Rev. Lett., 87, 16105 (2001)

25. E. Artacho, and F. Ynduráin, Phys. Rev. Lett. 62, 2491 (1989); E. Artacho, and F. Ynduráin, Phys. Rev. B 42, 11310 (1990)

26. R. G. Parr, W. Yang, Density Functional Theory, Oxford University Press, 1989

27. J. A. Pople.; P. M. W. Gill; N. C. Handy, Intl. J. Quantum. Chem., 56, 303 (1995)

28. C. Lee, W. Yang, R. G. Parr, Phys. Rev. B 37, 785 (1988); A. D. Becke, J. Chem. Phys. 98, $5648(1993)$ 
29. W. J. Hehre, R. Ditchfield, J. A. Pople, J. Chem. Phys. 56, 2257 (1972); M. M. Francl, W. J. Pietro, W. J. Hehre, J. S. Binkley, M. S. Gordon, D. J. DeFrees, J. A. Pople, J. Chem. Phys, 77, $3654(1982)$

30. T. Van Voorhis and M. Head-Gordon, Mol. Phys. 100, 1713 (2002); B.D. Dunietz, T. Van Voorhis and M. Head-Gordon, J. Theor. Comput. Chem. 1, 255 (2002).

31. R. Seeger and J. A. Pople, J. Chem. Phys. 66, 3045 (1977)

32. J. Kong, C. A. White, A. I. Krylov, D. Sherrill, R. D. Adamson, T. R. Furlani, M. S. Lee, A. M. Lee, S. R. Gwaltney, T. R. Adams, C. Ochsenfeld, A. T. B. Gilbert, G. S. Kedziora, V. A. Rassolov, D. R. Maurice, N. Nair, Y. H. Shao, N. A. Besley, P. E. Maslen, J. P. Dombroski, H. Daschel, W. M. Zhang, P. P. Korambath, J. Baker, E. F. C. Byrd, T. V. Voorhis, M. Oumi, S. Hirata, C. P. Hsu, N. Ishikawa, J. Florian, A. Warshel, B. G. Johnson, P. M. W. Gill, M. HeadGordon, J. A. Pople, J. Comp. Chem., 21, 1532 (2000)

33. M. W. Schmidt, K. K. Baldridge, J. A. Boatz, S. T. Elbert, M. S. Gordon, J. H. Jensen, S. Koseki, N. Matsunaga, K. A. Nguyen, S. Su, T. L. Windus, M. Dupuis, and J. A. Montgomery, Jr., J. Comp. Chem. 14, 1347 (1993)

34. Use of a bigger basis, namely 6-311+G(2df), for 1- and 2-dimer clusters consistently found a spin-restricted B3LYP solution to be unstable for symmetric dimers, although it slightly reduced the degree of symmetry breaking. With this basis, $<S^{2}>$ and spin densities at the dimer Si atoms for 1- and 2-dimer clusters were calculated to be 0.08 and \pm 0.21 , and 0.77 and \pm 0.45 , respectively. Also see Table 2 for comparison with 6-31G(d) results. 


\section{Figure Captions}

Figure 1. $\mathrm{Si}_{27} \mathrm{H}_{24}$ 4-dimer cluster models (Bigger balls are silicon and smaller balls are hydrogen), the largest cluster model used in this study. Upper panel shows the symmetric minimum, and lower panel the buckled minimum, both optimized at the (U)B3LYP/6-31G(d) level.

Figure 2. In this work, the buckling angle is defined as a dot product between two vectors, one of which is the dimer vector connecting atoms 3 and 4 and the other is the vector connecting the mid point between atoms 1 and 5 and another mid point between 2 and 6 . 
Table 1. Buckling angles, spin densities at middle dimer sites, $\left\langle S^{2}>\right.$ values, and middle dimer bond lengths for the DFT optimized buckled structures. The buckled structures do not have spin-unrestricted solutions except for the 1-dimer cluster, which is nearly identical to the symmetric structure.

\begin{tabular}{lccccc}
\hline \hline & Buckling & & & Dimer bond & $\Delta \mathrm{E}(\mathrm{R} \rightarrow \mathrm{U})^{\mathrm{a}}$ \\
Buckled & angle $\left(^{\circ}\right)$ & Spin densities & $<S^{2}>$ & length $(\AA)$ & $(\mathrm{kcal} / \mathrm{mol})$ \\
\hline 1-Dimer & 0.4 & \pm 0.41 & 0.17 & 2.238 & -0.13 \\
2-Dimer & 13.1 & 0.00 & 0.00 & 2.266 & 0 \\
3-Dimer & 15.4 & 0.00 & 0.00 & 2.298 & 0 \\
4-Dimer & 15.3 & 0.00 & 0.00 & 2.297 & 0 \\
\hline \hline
\end{tabular}

${ }^{a}$ Energy difference between the spin-restricted and -unrestricted solutions. Negative value means that there is a lower energy spin-unrestricted solution, whereas zero means that the spin-restricted solution is stable. 
Table 2. Spin densities at middle dimer sites, $\left\langle S^{2}>\right.$ values, and middle dimer bond lengths for the symmetric structures, optimized with unrestricted DFT.

\begin{tabular}{|c|c|c|c|c|}
\hline Symmetric & Spin densities & $<S^{2}>$ & $\begin{array}{l}\text { Dimer bond } \\
\text { length }(\AA)\end{array}$ & $\begin{array}{r}\Delta \mathrm{E}(\mathrm{R} \rightarrow \mathrm{U})^{\mathrm{b}} \\
(\mathrm{kcal} / \mathrm{mol})\end{array}$ \\
\hline 1-Dimer ${ }^{\mathrm{a}}$ & \pm 0.41 & 0.18 & 2.239 & -0.16 \\
\hline 2-Dimer ${ }^{\mathrm{a}}$ & \pm 0.60 & 0.81 & 2.262 & -2.01 \\
\hline 3-Dimer & \pm 0.67 & 1.27 & 2.279 & -3.39 \\
\hline 4-Dimer & \pm 0.65 & 1.77 & 2.275 & -2.77 \\
\hline
\end{tabular}

${ }^{\mathrm{a}}$ See also reference 34 .

${ }^{b}$ Energy difference between the spin-restricted and -unrestricted solutions (Note that it is not the energy difference per dimer). Negative value means that there is a lower energy spin-unrestricted solution. 
Table 3. Relative energy difference $\left(\Delta \mathrm{E}=\mathrm{E}_{\mathrm{sym}}-\mathrm{E}_{\text {buck }}\right)$ per dimer between the buckled $\left(\mathrm{E}_{\text {buck }}\right)$ and symmetric $\left(E_{\text {sym }}\right)$ minima obtained at the $(\mathrm{U}) \mathrm{B} 3 \mathrm{LYP} / 6-31 \mathrm{G}(\mathrm{d})$ level. Negative values mean that the symmetric structure is lower in energy than the buckled structure.

$\Delta \mathrm{E} / \mathrm{dimer}(\mathrm{kcal} / \mathrm{mol})$

\begin{tabular}{|c|c|c|c|c|c|}
\hline & $\begin{array}{l}\text { (U)B3LYP } \\
\text { (This work) }^{\mathrm{a}}\end{array}$ & 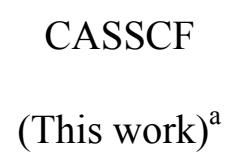 & $\begin{array}{c}\text { MRMP2 } \\
\text { (This work) }^{\mathrm{a}}\end{array}$ & $\begin{array}{c}\text { BPW91 } \\
(\text { reference } 17)^{b, d}\end{array}$ & $\begin{array}{c}\text { DMC } \\
(\text { reference } 24)^{b}\end{array}$ \\
\hline 1-Dimer & 0.05 & -0.02 & -0.01 & 0.85 & \\
\hline 2-Dimer & 0.74 & $-6.71^{\mathrm{c}}$ & $-3.35^{\mathrm{c}}$ & 3.76 & 0.69 \\
\hline 3-Dimer & 1.40 & -6.54 & -4.01 & 5.28 & 2.54 \\
\hline 4-Dimer & 1.54 & & & 5.81 & \\
\hline
\end{tabular}

${ }^{\mathrm{a}}$ Geometries obtained from spin-unrestricted DFT were used for the relative energy calculations in this work. ${ }^{b}$ Note that these relative energies used symmetric geometries from restricted DFT calculations, which are likely to be unstable. ${ }^{c}$ Use of a bigger basis (i.e., 6-31+G(3df) for the dimer and 6-31G(d) for the rest of a cluster) yields a $\Delta \mathrm{E} /$ dimer of -5.34 and $-3.09 \mathrm{kcal} / \mathrm{mol}$ at the $\mathrm{CASSCF}(4,4)$ and MRMP2(4,4) level, respectively, essentially the same result as with a 6-31G(d) basis for the entire cluster. ${ }^{\mathrm{d}} \mathrm{SBK}$ effective core potential (ECP) basis was used. For a 2-dimer cluster, use of all-electron 6$311 \mathrm{G}(2 \mathrm{~d})$ yielded a $\Delta \mathrm{E} /$ dimer of $2.85 \mathrm{kcal} / \mathrm{mol}$, slightly smaller than using an ECP basis $(3.76 \mathrm{kcal} / \mathrm{mol})$. 

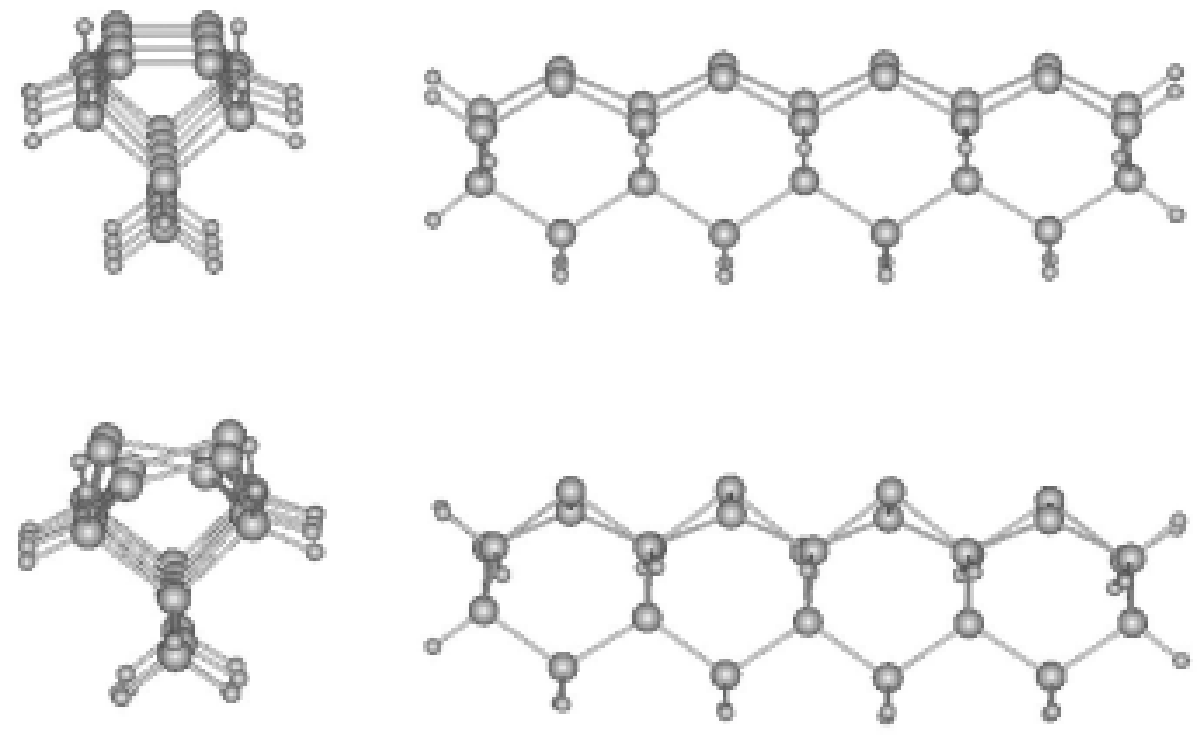

Figure 1 


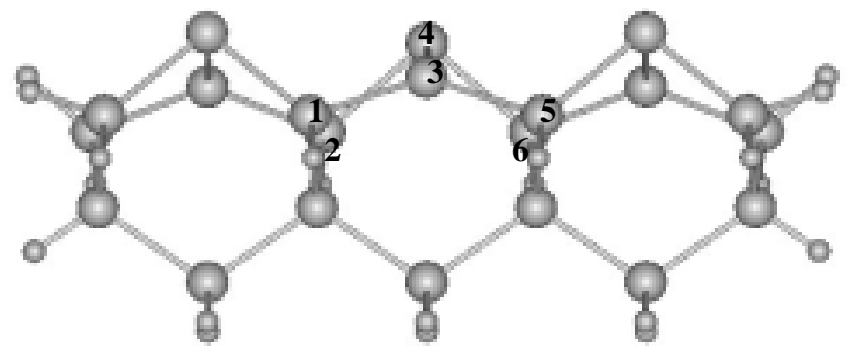

Figure 2 